\section{OP17 \#ETHNIC AND SOCIAL VARIATION IN CAUSE-SPECIFIC INFANT MORTALITY OF PRETERM BABIES, ENGLAND AND WALES 2006-2012: NATIONAL BIRTH COHORT WITH ROUTINE DATA}

ME Kroll*, MA Quigley, JJ Kurinczuk. National Perinatal Epidemiology Unit, University of Oxford, Oxford, UK

\subsection{6/jech-2018-SSMabstracts.17}

Background Inequalities in infant mortality in England and Wales remain of concern. Patterns of risk for preterm babies (over 50\% of infant deaths) are poorly understood, and may differ by cause of death and degree of prematurity. We aimed to describe ethnic and social variation in cause-specific infant mortality of preterm babies by level of prematurity, and examine the extent to which disadvantage might mediate ethnic variation, or ethnicity explain social variation.

Methods The Office for National Statistics linked birth and death registrations with other routine data for singleton live births at gestational age 24-36 weeks in England and Wales 2006-2012. Within three gestation categories (24-27, 28-31, 32-36 weeks), we fitted Poisson regression models with robust standard errors (adjusted for potential confounding by gestation week, birth year, and gender), relating ethnicity (9 groups) and/or area deprivation (IMD quintiles) to risk of infant death from congenital anomalies, immaturity-related conditions, or all other causes.

Results There were 256142 births and 6480 deaths (26\% from congenital anomalies, 52\% immaturity-related).

Among 24-27 week births (5\% of preterm babies, but $47 \%$ of those who died in infancy), infants of all minority ethnic groups had lower risk of immaturity-related death than White British, the lowest rate ratios being 0.65 (95\% CI 0.51 to $0.83)$ for Black Caribbean, 0.76 (0.66-0.87) for Black African, and $0.76(0.61-0.94)$ for Indian.

Among 32-36 week births, infants of all minority groups had higher risk of death from congenital anomalies than White British, the highest rate ratios being 4.86 (4.10-5.77) for Pakistani, $3.12(2.28-4.28)$ for Bangladeshi, and 2.21 (1.71-2.87) for Black African. Risks of death from congenital anomalies and 'other' causes increased with disadvantage, rate ratios comparing the most with the least deprived quintile being respectively $2.01(1.60-2.51)$ (attenuated to 1.54 (1.22$1.94)$ by adjustment for ethnicity) and 2.07 (1.57-2.73).

Except for social variation in death from congenital anomalies, adjusting disadvantage models for ethnicity, or vice versa, made little difference.

Conclusion Ethnic variation in infant mortality following preterm birth is driven by contrasting patterns of death from immaturity-related conditions in 24-27 week babies, and congenital anomalies in $32-36$ week babies. Social variation is driven by deaths from congenital anomalies (perhaps in part explained by ethnicity) and 'other' causes. We found no evidence that ethnic variation was mediated by disadvantage. Further work is needed to establish reasons for ethnic variation in death from congenital anomalies. Future research should examine biological causes of very preterm birth.

\section{OP18 EVALUATING THE IMPACT OF THE ENGLISH HEALTH INEQUALITIES STRATEGY ON SOCIOECONOMIC INEQUALITIES IN THE REGIONAL INFANT MORTALITY RATE}

${ }^{1}$ TJ Robinson*, ${ }^{2}$ B Barr, ${ }^{1} \mathrm{HW}$ Brown, ${ }^{3} \mathrm{~L}$ Fraser, ${ }^{4} \mathrm{P}$ Norman, ${ }^{1} \mathrm{C}$ Bambra. ${ }^{1}$ Institue of Health and Society, Newcastle University, Newcastle upon Tyne, UK; ${ }^{2}$ Institute of Psychology Health and Society, University of Liverpool, Liverpool, UK; ${ }^{3}$ Department of Health Sciences, University of York, York, UK; ${ }^{4}$ School of Geography, University of Leeds, Leeds, UK

\subsection{6/jech-2018-SSMabstracts. 18}

Background Following the election of a Labour government in 1997 on a mandate that included a commitment to reducing health inequalities (HI) and implementing evidence-based policy, the UK became the first European country in which policy-makers systematically and explicitly attempted to reduce inequalities in health. One explicit target was to reduce socioeconomic disparities in infant mortality. However, it remains unclear whether the HI strategy had an impact on socioeconomic geographical inequalities in the infant mortality rate (IMR). We investigated whether the period of the HI strategy was associated with a change in the difference in the IMR between the most disadvantaged local authorities and the country as a whole compared with trends before and after the strategy.

Methods We used data from the UK data achieve and the ONS on the annual number of births and infant deaths in 323 local authority areas across England from 1983-2016. We initially calculated the IMR in the most deprived group of local authorities and the rest of England from 1983 to 2016, and the relative and absolute differences between these groups to investigate trends in inequalities before, during and after the strategy was introduced. We then used the panel of data to estimate segmented fixed effects regression models, including linear spline terms for time with two breakpoints at the beginning and end of the strategy period and an interaction term between the time trend and a dummy variable indicating the deprived groups of local authorities.

Results Prelimary results show that before the HI strategy, the relative gap in the IMR increased at a rate of $1.3 \%$ each year $(95 \%$ confidence interval $0.8 \%$ to $1.8 \%)$. During the strategy period this trend reversed and the relative gap in the IMR decreased at a rate of $1.7 \%$ each year $(95 \%$ confidence interval $0.7 \%$ to $2.8 \%$ ). Since the end of the policy period, the relative gap in the IMR has continued to decrease (at a rate of $1.3 \%$ each year), however this change was found to not be statistically significant (95\% CI $-3.7 \%$ to $1.1 \%)$.

Conclusion The English HI strategy was associated with a small decline in relative geographical inequalities in the IMR, reversing a previously increasing trend. This result was found to be statistically significant $(p<0.001)$. Future work will test the robustness of these results, through the use of different econometric methods (including Poisson regression models) and the inclusion of a number of controlling variables. 